\title{
The Role of Minimally Invasive Spine Surgery
}

\author{
Rully Hanafi Dahlan ${ }^{1}$, Sevline Estethia Ompusunggu ${ }^{1}$, Christian Ade $^{1}$, Ayu Iswandari Raharjo ${ }^{1}$ \\ ${ }^{1}$ Spine Peripheral and Pain division, Department of Neurosurgery Padjadjaran University,Bandung, \\ Indonesia
}

\begin{abstract}
Introduction :In the past half of century, the spinal surgery techniques has advanced significantly. Along with the improvement and various of techniques and technologies in general, there has been a big movement to reduce the morbidity of surgery.

Case review : As opposed to open spine surgery, minimally invasive surgical approaches can be faster, safer and require less recovery time. The minimally invasive spine surgery also need to make an efficient target of surgery. The roots in minimally invasive spine surgery (MISS) are based primarily on technique modifications.

Discussion: The Williams microdiscectomy, described in 1978, revolutionized MISS by starting the evolution of lumbar discectomy from an open surgery through a 6-inch incision to a microsurgical approach through as small an opening as possible.

Conclusion :We don't use the MISS technique when the extension of tumor is more than 2 levels; extension of the tumor is $20 \%$ longer than diameter of largest retractor; the tumor $>3 \mathrm{~cm}$ for interlaminary approach, the wide durotomy is needed; and also the case with intramedullary tumor with $80 \%$ extention, from left to the right side; en bloc as the the goal of surgery for extradural tumor
\end{abstract}

Keyword: Spine Surgery, Minimally Invasive, Spine

\section{Introduction}

In the past half of century, the spinal surgery techniques has advanced significantly. Along with the improvement and various of techniques and technologies in general, there has been a big movement to reduce the morbidity of surgery. As opposed to open spine surgery, minimally invasive surgical approaches can be faster, safer and require less recovery time. The minimally invasive spine surgery also need to make an efficient target of surgery. The development of spine surgical techniques has been change, from the open surgery to mini-open surgery, and then to be a minimally invasive spine surgery (Figure 1).

In modern medicine, there are few advances that have had a more meaningful impact on patient outcomes than minimally invasive spine procedures. Patients presenting with painful radiculopathies and neural compression can be treated successfully with minimal access procedures and sent home the same day.[1]

*Corresponding author at: Spine peripheral and Pain division, Department of Neurosurgery, Hasan Sadikin Hospital, Padjadjaran University,Bandung, Indonesia 
The roots in minimally invasive spine surgery (MISS) are based primarily on technique modifications. The Williams microdiscectomy, described in 1978, revolutionized MISS by starting the evolution of lumbar discectomy from an open surgery through a 6-inch incision to a microsurgical approach through as small an opening as possible. The Wiltse approach, described in 1968, was revolutionary insomuch as the dissection in the spine was achieved between muscular planes, as opposed to through the softtissue envelope or the subperiosteal plane. Subsequent developments have heavily leveraged new technologies, including enhanced retraction, fixation, biologics, visualization, monitoring, and navigation, further disrupting the landscape [1].

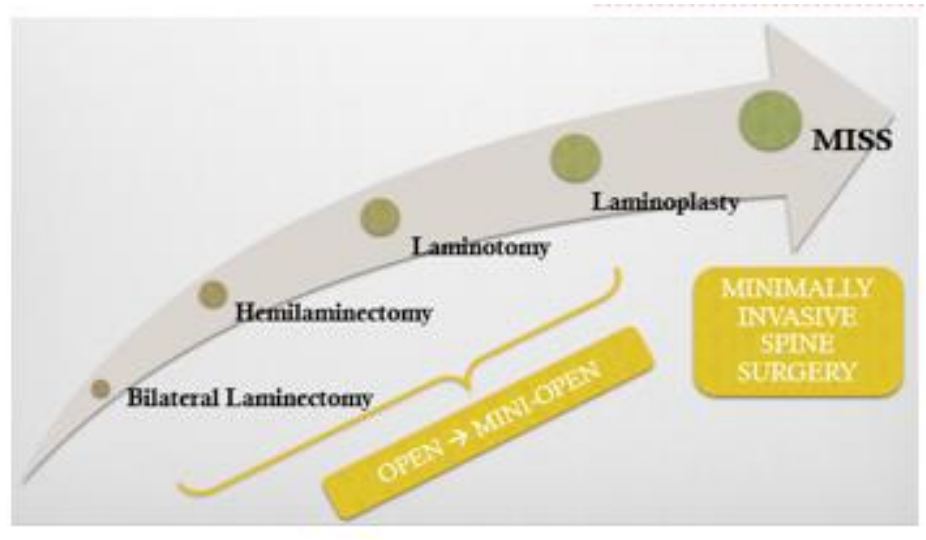

Figure 1. Surgical Technique Development

\section{Case Review}

There is some application of MISS for anterior approach surgery, such as: Discectomy, Total Disc Replacement, Fractures, Tumors, and Decompression (For Cervical Spine); Disc Herniations (For Thoracic Spine); Mini ALIF, Nucleus Replacement, Total Disc Replacement, Fractures, Tumors, and Decompression (For Lumbar Spine). And also for posterior approach surgery: Decompression (For Cervical Spine); Costotranversectomy (For Thoracic Spine); Disc Herniation and Exicions, Spinal Stenosis, Foraminal Stenosis, and also PLIF/TLIF procedures (For Lumbar Spine). In between of open surgery and minimally invasive spine surgery (MISS), there are some superiorities on both technique [1].

Open surgery has a long midline incision compared to MISS have a small incision compared to long midline incision in open surgery, that cosmetically vicious than MISS. Open surgery has a risk of massive bleeding due to bilateral subperiosteal muscle stripping, and also a risk of posterior spinal elements disruption. But this open surgery techniques, made a spine surgeon enable to perform laminectomy extend to the 
level above and below the spinal tumor. This open surgical surgery made an exposure and the surgical manipulation of the tumor is easier [1].

Minimally invasive spine surgery (MISS) reduce the post operative pain due to less tissue injury, minimalized the blood loss and time, reduce the possibility risk of CSF leakage by decreasing of death space. MISS also made a recovery time to do daily activity quicker. This technique avoid a need of fusion and reduce the risk of postlaminectomy instability. The conclusion is, this MISS technique minimalized the post operative complication [1].

In the spine surgery, the principle are spinal decompression, and stabilization. In the recovery phase, the patient need a restoration and also rehabilitazion. In general, the degenerative spine disease is one of the most common spinal cases. The knowledge about when we do surgery, or do no surgery, that's such a complex thing. The indication and also patient selection is an important thing. MISS play a role of surgery, to get a minimal post operative complication [1].

The decision-making challenges of surgically treating spinal neoplasms are many and include the individual patient's symptom profile, the nature and morphology of the tumor(s), presence of multiple or peripheral metastases, neurologic or pending neurologic status (spinal cord compression), resultant spinal deformities or instabilities, adjuvant radiation or chemotherapies, and expected life span. The minimally invasive surgical treatment of spinal tumors has historically been considered oxymoronic, as exposures that allow for adequate tumor exposure and resectioning were, by nature, open exposure approaches. Less-invasive exposures were long considered inadequate in managing a variety of tumors, especially when en bloc resection is required. With the advent of stereotactic radiotherapy, the development and proliferation of endoscopic and mini-open approaches, and the paradigm shifts in spinal oncology thinking, a new era of individualized multidisciplinary approaches has emerged, minimizing morbidity and maximizing effectiveness. [1][2]

Several less-invasive approaches for spine tumor removal are currently being used and can be broadly categorized as thoracoscopic, mini-open anterior, and miniopen posterior approaches. Each approach has benefits and drawbacks and should be tailored to the individual needs of the patient. Because these patients usually undergo these procedures for quality of life (prevention of paralysis, maintenance of continence, and pain control), minimizing the morbidity from the surgery is paramount. Moreover, faster wound healing is desirable to allow for early 
postoperative radiation or chemotherapy. The goal of a less-invasive approach is to try to achieve these two goals, yet at the same time still accomplish the same surgical result [2].

The pre operative clinical judgment or patient selection is important. We need to predict the tumor behaviors wich is primer or secondary tumor. Bone erotion, multilevel involvement or any foraminal extension or the need of post operative fusion are important. MISS is technology dependant surgery. The advance tools and instrumentation are needed; endoscope system, tubular retractor (expandable or not expandable), microscope, and fluoroscopy imaging. The step of learning curve, first the surgeon need to learn or develop his spatial and 3D orientation in 2D monitor, and also the knowledge about anatomical orientation with manipulation in limited working space, etc.

In the thoracic spinal tumor cases, we need to know that the thoracic region is one of the most difficult region to access. The anterior approach is more challenging surgery due to the significant mortality and morbity with involvement of cardiopulmonary system, and we need to thoracic surgeon to perform thoracotomy before. But, with MISS technique, thoracosopy made a shorter hospitalization, decreased of blood loss, and shorter operating time. In the lumbar spinal tumor cases, anterior approach still more challenging surgery. The posterior approach lumbar spine surgery, we need to learn about an unique lumbar characteristic of lamina in lumbar vertebrae (wide wedge-shaped interlaminar space; widest at the center) that could be the consideration to do an interlaminar approach with tubular system or transpinous approach (Figure 2,3) [2].
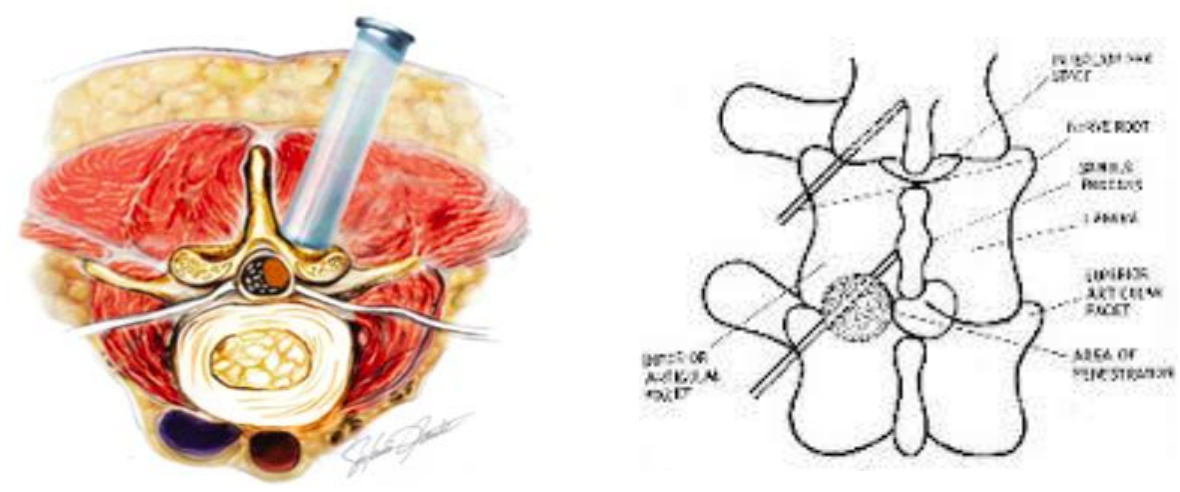

Figure 2. Interlaminar approach technique 


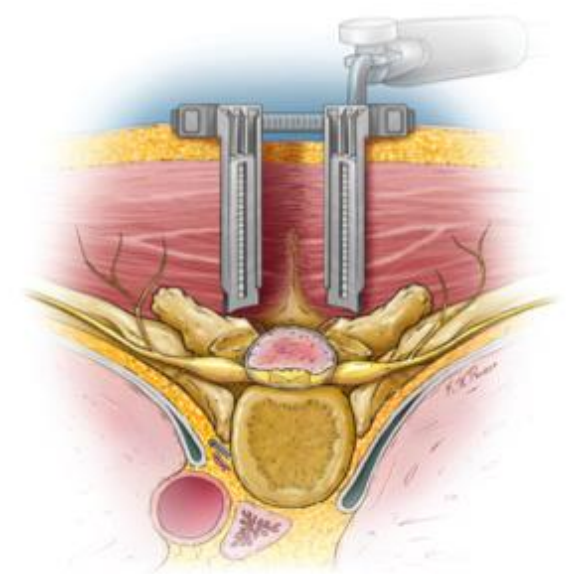

Figure 3. Trans-spinous approach

\section{Discussion}

The tumor characteristic recommendation for MISS surgery; the tumor size less than $2 \mathrm{~cm}$ at the long axis diameter, the cyst tumor consistency makes it possible to remove eventhought the size is more than $2 \mathrm{~cm}$. If there is the firm involvement of duramater, the large durotomy is needed, and increase the risk of CSF leakage after surgery or there is an extraforaminal extention; those cases are not best for MISS technique. The multilevel involvement, 2 levels still safe for MISS [2].

In 2014, Zhong et al., made a study of 122 patient with intradural extramedullary tumors. They compared the treatment results in the different surgeries of spinal intradural extramedullary tumor. The study retrospectively reviewed 122 patients. The minimally invasive $\backslash$ surgery (MIS) group was divided into Group A (hemilaminectomy + tumor microscopic excision) and Group B (laminectomy + tumor microscopic excision + pedicle screw fixation). Meanwhile, the non-MIS group was divided into Group C (hemilaminectomy + tumor excision), Group D (laminectomy + tumor excision), and Group E (laminectomy + tumor excision + pedicle screw fixation). In order to study postoperative spinal stability, we simultaneously divided all of the subjects into three categories, namely Group HE: hemilaminectomy + tumor excision; Group LE: laminectomy + tumor excision; and Group LEPSF: laminectomy + tumor excision + pedicle screw fixation. And the results, The MIS group exhibited fewer postoperative complications $(\mathrm{p}<0.05)$, better short-term clinical efficacy $(\mathrm{p}<0.05)$ and less non-surgical cost $(\mathrm{p}<0.05)$ than in non-MIS group. The rate of postoperative spinal instability in hemilaminectomy was lower than in laminectomy in a single spinal segment $(\mathrm{p}<0.05)$. The rate of postoperative spinal instability in laminectomy + pedicle screw fixation was lower than in hemilaminectomy and laminectomy in two or more spinal segments $(\mathrm{p}<0.05)$. They conclude, in the case of appropriate surgical indications, 
minimally invasive surgery for intradural extramedullary tumor is a useful method that can successfully produce good clinical results and reduce non-surgical cost. In addition, pedicle screw fixation helps avoid spinal postoperative instability (figure 4) [3].

\begin{tabular}{|c|c|c|c|c|c|c|}
\hline Groups & $\mathrm{N}$ & $\begin{array}{l}\text { Operating } \\
\text { time(min) }\end{array}$ & $\begin{array}{l}\text { Intraoperativeblood } \\
\text { loss }(\mathrm{ml})\end{array}$ & $\begin{array}{l}\text { Resectionrate } \\
(\%)\end{array}$ & $\begin{array}{l}\text { Duration ofhospital } \\
\text { stay(d) }\end{array}$ & Complications \\
\hline MIS group & 53 & $270.00 \pm 147.99^{\star}$ & $307.14 \pm 185.13^{\star}$ & $77.36^{\mathbf{\Lambda}}$ & $21.88 \pm 10.15^{\star}$ & $2^{\star}$ \\
\hline $\begin{array}{l}\text { Non- } \\
\text { MISgroup }\end{array}$ & 69 & $173.26 \pm 81.36$ & $540.08 \pm 282.15$ & 72.46 & $26.42 \pm 11.60$ & 12 \\
\hline
\end{tabular}

This table shows the results for operating time, intraoperative blood loss, resection rate, duration of hospital stay, and complications in the MIS and Non-MIS group. "Resection rate" means the percentage of the cases of total resection of tumor. Data are expressed as the mean \pm standard deviation.

$\star p<0.05$ vs. MIS group;

$\star p>0.05$ vs. Non-MIS group.

Figure 4. Summary of the results for operating time, intraoperative blood loss, resection rate, duration of hospital stay, and complications.

We don't use the MISS technique when the extension of tumor is more than 2 levels; extension of the tumor is $20 \%$ longer than diameter of largest retractor; the tumor $>3 \mathrm{~cm}$ for interlaminary approach, the wide durotomy is needed; and also the case with intramedullary tumor with $80 \%$ extention, from left to the right side; en bloc as the the goal of surgery for extradural tumor; Tokuhashi score $>11$; transverse diameter of extramedullary tumor $>75 \%$ spinal canal diameter; if there is extra foraminal extension, the highly need for fusion; cases with tumor with wide ventral attachment and wide ventral component; and the highly need for wide decompression. $[3][4]$

The high risk factor of MISS surgery are inadequate training/ lack of maturity; an insufficient knowledge of anatomy and experience of surgery; wrong choice of patient, approach, and technique; overzealousness; lack of anticipation; wrong decision making; and technical reasons. [3][4]

\section{Conclusion}

To minimalized the complication, we need to have a good learning curves and also experienced of surgery. It is easier to stay out of trouble than get out trouble, so we need to know our costumer. Anticipation comes by knowing the patient's history 
and investigations. So, the patient selection, learning curves, and appropriate technique, technology and goals are important. 


\section{References}

[1]. Härtl, Roger; Korge, Andreas: 2014. Minimally Invasive Spine Surgery techniques, Evidence, and Controversies. AOSpine MISS Curriculum Task Force 2018-2020.

[2]. Iacoangeli M, Gladi M, Di Rienzo A, Dobran M, Alvaro L, et al. (2015) Minimally invasive surgery for benign intradural extramedullary spinal meningiomas: experience of a single institution in a cohort of elderly patients and review of the literature. Clinical interventions in aging. 7: 557-564.

[3]. Shaohui Zong, et al. Treatment Results in the Different Surgery of Intradural Extramedullary Tumor of 122 Cases. 2014.

[4]. Yasuaki Tokuhashi, Hiroshi Uei, Masashi Oshima, and Yasumitsu Ajiro. 2014. Scoring system for prediction of metastatic spine tumor prognosis 